\title{
'What are the perceived implications, if any, for non-IAPT therapists working in an IAPT service?'
}

\begin{abstract}
The research aimed to investigate the national Improving Access to Psychological Therapies (IAPT) programme, from the perspective of 'non-IAPT' counsellors and psychotherapists working within IAPT services. Semi-structured interviews were carried out with therapists who were currently working within IAPT services, but had no formal 'IAPT-compliant' or 'IAPT-accredited' training. Foucauldian discourse analysis was used to analyse interview transcriptions and the study examined the various discourses used by participants to speak about IAPT. Findings indicated that participants drew upon dominant discourses in attempts to fit in or belong, yet paradoxically, the way in which the discourses were used seemed to create subject positions that were outside to, or excluded from, the IAPT services they worked at. IAPT and non-IAPT discourses emerged simultaneously - a possible indication of the discursive junction where different ideologies were in conflict with one another. Implications of the power relations that may be operating within this setting are discussed and suggestions made for further research.
\end{abstract}

Keywords: Improving Access to Psychological Therapies (IAPT), discourse analysis, Foucault, National Institute of Health and Clinical Excellence (NICE), power relations, subject positions

Word Count $=\mathbf{6 , 3 8 2}$ words excl. Abstract and References 


\section{Introduction}

This qualitative study aimed to investigate the implications of the national Improving Access to Psychological Therapies (IAPT) programme, from the perspective of 'nonIAPT' counsellors and psychotherapists working within IAPT services. In order to explain what is meant here by the term 'non-IAPT' therapists, it becomes necessary to give a brief outline of the history of the IAPT programme.

'Depression' and 'anxiety' have been identified as the 'two most common mental disorders' (CMDs) in the United Kingdom (HSCIC, 2009) and in 2005 a report presented to the British government (Layard, 2005) put forward a strong economicbased argument for the treatment of those suffering from these 'disorders'. The Labour government responded with substantial investment in primary care mental health services and 'IAPT' was conceived as a way of helping National Health Service (NHS) patients gain easier access to psychological treatment.

Clark (2011: 318) views the IAPT programme as having 'its roots in a wide range of clinical and policy developments'. One of these developments, considered relevant to this particular study, involves the 'National Institute for Health and Clinical Excellence' (NICE). This organisation 'provides national guidance and advice to improve health and social care' (NICE, 2013), including which psychological therapies should be provided by the NHS. There continues to be much debate around the fact that services such as IAPT are governed by the NICE guidelines (Guy et al, 2012: 25) and NICE has been criticised for only recommending those modalities of psychological therapy considered to be 'evidence based', with 'minimal, or no, recommendations for counselling or counsellor based therapies' (Cooper, 2011: 2). 
When the first phase of the programme was implemented between 2008 and 2011, it followed a national curriculum that focused on Cognitive Behavioural Therapy (CBT) and the training of psychological therapists specifically in this approach, as this was where the manpower shortage was considered the greatest (Clark et al, 2009: 910). In its more recent phase since 2011, the IAPT curriculum has expanded further to include four additional 'IAPT-compliant' modalities in counselling and psychotherapy and these are: CfD (Counselling for Depression), IPT (Interpersonal Therapy), DIT (Dynamic Interpersonal Therapy) and CofD (Couple Therapy for Depression), (IAPT, 2011).

But this is where it gets complicated. IAPT services also include counsellors and psychotherapists who are neither trained in CBT, nor any of these four 'compliant' modalities. This seems to be due, in part, to the 'contracting out' process where funding is allocated to external counselling agencies through a competitive tendering process. The motivation for this study has emerged from the experience of one of the researchers, who found herself working for such a counselling agency, when the agency was awarded the IAPT contract by a particular Clinical Commissioning Group (CCG). All counsellors working at the agency were required to take on an additional caseload of four IAPT clients in addition to their existing client work and, in order to fit in with IAPT guidelines, certain ways of working with clients were implemented, including Patient Health Questionnaires (PHQ-9), Generalised Anxiety Disorder Assessment (GAD-7), Outcome Measures (CORE-10) and compulsory use of a computer system called 'IAPTus'. Such different ways of working with clients raised questions for the researcher about the compatibility of her core theory (an integrative psychotherapy training) with these assessments and measures. An interest arose in finding and interviewing other 'non-IAPT' therapists 
who had found themselves in a similar situation, working in an IAPT service, and the research question was formulated as: 'What are the perceived implications, if any, for non-IAPT therapists working in an IAPT service?' Such a question aimed to explore the possible perceived implications for therapists, but also allowed for the possibility that perhaps there were no implications.

\section{Method}

\section{Design}

A literature review indicated that, although there was much quantitative research about the function and performance of the IAPT programme, there seemed to be a gap in the availability of qualitative research on the subject. The area where the literature, and previous research studies, seemed to be most lacking was that regarding therapists' experience of working within IAPT services, in particular where therapists had not completed an official IAPT-compliant or -accredited training. According to Silverman (2006: 59), '[a] dependence on purely quantitative methods may neglect the social and cultural construction of the "variables" which quantitative research seeks to correlate' and therefore it was decided that a qualitative research method would therefore be more appropriate for the research question. Qualitative inquiry ignores fixed assumptions and 'holds the promise of discovery, of generating new insights into old problems, and producing nuanced accounts that do justice to the experience of all those participating in the research' (McLeod, 2001: 1).

Although several qualitative research methods were originally considered, Foucauldian Discourse Analysis was selected for a number of reasons. According to Parker et al (1995: 10), 'Foucault's work has been invaluable in drawing attention to the way language is organised around different systems of meaning which offer 
positions of power to certain categories of people while disempowering others. These systems of meaning are discourses'. It was considered that this version of Discourse Analysis might offer exploration of the language that participants used to talk about IAPT. Additionally, as the literature review had raised questions about the relationship between institutional practices and power, Foucauldian Discourse Analysis seemed a suitable choice as it has been described as aiming to 'explore power relations from a critical standpoint in an attempt to make sense of the social world by providing new critical insights' (Morgan, 2010: 1).

For Foucault (1972) it would be too simplistic to only look at power as a force possessed by or emanating from a sovereign or state, but rather he would see it as something that already exists in all human relations. According to Sarup (1993: 74), Foucault is rejecting of analyses that want to locate the origin of power within a structure or institution, in favour of questions that might uncover 'the processes by which subjects are constituted as effects of power'.

This view might sound as if such a research method is not interested in subjectivity, yet Heenan (in Loewenthal \& Winter, 2006: 184) argues that discourse analysts are interested in subjectivity and self-awareness, but their main focus is 'in how subjects are discursively positioned by language within wider social discourses'.

It was hoped that such a qualitative research method might be helpful in opening up a dialogue about this topic in the psychotherapeutic profession.

\section{Participants}

The need for such a dialogue was highlighted when a request to recruit participants through the researcher's placement was rejected. The managing director of the agency did not respond to any attempts at contact over the period of a month and 
finally a well-meaning supervisor responded: 'Can you understand why you can't ask that question here?' He said that asking such questions might be perceived as 'threatening' by the organisation. Ethical approval was therefore sought to recruit individual participants outside of this placement, and participants were recruited externally through professional networks by word of mouth and email. The email outlined the research question and overall research project and set out the eligibility criteria that participants were to be working as counsellors or psychotherapists within an IAPT counselling service, but were not to have completed an IAPT approved or accredited training. Ultimately, five participants were interviewed. These participants came from different approaches and training backgrounds, identifying themselves as: Existential (2), Post-Existential (1), Person-Centred (1) and Integrative (1). All were currently involved in post-graduate training programmes at either MSc year or PsychD level.

\section{Data collection and analysis}

During the semi-structured interviews, participants were asked open-ended questions and the interviews were tape recorded. A qualitative research interview 'is always semi-structured because it invariably carries the traces of patterns of power that hold things in place and it reveals an interviewee's... creative abilities to refuse and resist what a researcher wants to happen' (Parker, 2005: 53). It is by examining the intersection of both the researcher and participant's agendas that 'we open up an individual account into something spoken in a context and something spoken against it' (2005: 54).

Willig (2008: 115-123) offers six steps or 'stages' on how to conduct a Foucauldian Discourse Analysis. Foucault (1994: 288) himself made it clear that he 
disliked prescription and once stated 'I take care not to dictate how things should be' and therefore a Foucauldian Discourse Analysis is regarded as an 'approach or stance rather than a method' (McLeod 2011: 180). With this in mind, Willig's six stages were used as a guideline rather than a set of 'rules' to be adhered to and all relevant extracts were examined in terms of: Discursive Constructions, Discourses, Action Orientation, Positionings, Practice and Subjectivity (Willig, 2008).

\section{Results}

For the purposes of this study, the discursive object was identified as 'the IAPT service'. Once the discursive object and the discursive constructions of it had been identified and highlighted in the interview transcripts, the transcripts were then reread, and an attempt was made to locate these constructions within the wider discourses that were drawn on by the participants, and brief examples of these are given under subheadings below. The possible subject positions that could be taken up by participants, as a result of drawing on these discourses, were then considered, in conjunction with the resulting opportunities for action. Finally, brief speculation about what could be 'felt, thought and experienced' (Willig, 2008: 117) by participants from these various positions is discussed.

\section{Professional Discourse}

A professional discourse appeared to emerge in those instances where participants talked about the various IAPT services that they worked for as if they were large businesses or corporations. The following two extracts demonstrate how this 
discourse was used to describe how the gaining of experience in IAPT would lead to future employment opportunities and career progression:

....and so I took the opportunity [ ] I wanted experience in IAPT because I thought it would look good on my CV... (Anne).

...maybe I am doing it in order to get the experience to make myself more um (.) sellable (.) you know that I can perhaps get a job in the NHS one day because there are positions out there but I need a reference... (Claire).

For these two participants, the construction of IAPT as a powerful service provider drew on a professional discourse where being affiliated with the IAPT service on a 'CV' or through a 'reference' is viewed as advantageous. Through the use of this discourse both participants were offered subject positions of business interns or apprentices, where working for IAPT was spoken of as a career move, leading to 'experience' and resulting in favourable references and future employment.

There seemed to be a difference in the kinds of subjective experience made available to these participants through the use of this discourse. Whereas Anne appeared to take up the position of a free agent who made her own decisions, Claire took up a position of reluctantly complying. Later in the interview she said:

...I have to conform to this (.) there's a pressure for me to get this IAPT experience so I could actually just get more clients [ ] even though there's part of 
me that's a bit you know going against the grain here with regard to my training but (2) perhaps it's the wrong way to go? [stops and looks at the researcher]... (Claire).

A construction of 'IAPT experience' as something that she has to 'conform' to offers a subject position that undermines her freedom and implies that sacrifices have to be made to 'get more clients'. She then made attempts to repair what the interviewer might assume about her, by saying that perhaps this is the 'wrong way to go' and looked in silence for feedback. Potter (2004, in Silverman 2004: 200-221) refers to this as an 'uncertainty token' where a speaker invites the listener to minimise their 'stake' and 'interest' in what is being said. A possibility is that Claire was concerned that the interviewer might have thought she was being hypocritical. If feelings of hypocrisy were made available from within this ambivalent subject position, the use

of an uncertainty token could be interpreted as a position whereby such feelings could be avoided.

\section{Institutional Discourse}

The construction of IAPT as being governed by institutional rules and processes seemed to resonate with an institutional discourse. The use of this discourse appeared different to that of a professional discourse, in that it was more about a 'top-down' approach, where employees find themselves accountable to managers or supervisors in positions of authority. The following participant drew on this discourse to describe how weekly scores needed to be reported in supervision: 
...I have to report to my supervisor what the clients' CORE score is (.) she wants to know from week to week whether it's gone up or down... (Claire).

Through the use of an institutional discourse, Claire seemed to take up a subject position subordinate to that of her supervisor, having to 'report' her client's weekly progress in terms of the 'CORE questionnaire' scores. She went on to say:

...l also got a sense it's used to measure my performance [ ] they're measuring $\underline{\text { me }}$ and [voice goes up to a higher pitch] this can feel a bit /intrusive/... (Claire).

In this extract, IAPT is constructed as being able to monitor or measure her 'performance' through her clients' CORE questionnaires and there was a change in pitch to her voice when she described this sense of being measured as 'intrusive'. She appeared to become anxious, and it could be interpreted that the presence of anxious feelings had been made available by her taking up of the subject position of someone who is monitored or watched. There was not much room for any kind of action on her part, which may have served to legitimise feelings of powerlessness.

The following participant appeared to take up a far more subversive position as he drew on an institutional discourse to talk about an IAPT induction at his placement: 
...well the most interesting experience and it was um laughable and fascinating but also um Orwellian was seeing um this experienced CBT practitioner deliver our IAPT induction and it was like she was using [ ] Newspeak?... (Ben).

He appeared to be questioning the authority of the CBT practitioner and compared the way in which she talked to 'Newspeak'. This assumed a shared understanding between himself and the interviewer of the fictional language described by George Orwell (1949) in his well-known novel 'Nineteen Eighty-Four'. It could be interpreted that he alluded to this dystopian tale of government surveillance to convey his distrust of the CBT practitioner and her position of authority. He continued to describe the incident:

...I enjoyed the number of times I stuck my hand up and said I didn't know this or that acronym and it was almost constant and I could see she was getting pissed off and sometimes she didn't know what the acronym was it was great [laughs]... (Ben).

There appeared to be a great deal of resistance in his part to the induction process. It could be interpreted that such a subversive subject position might lead to feelings of alienation from the IAPT service, yet at the time of the interview Ben was still working for IAPT. There is a possibility that the CBT practitioner was experienced by Ben as someone 'laughable' to be ridiculed because she was seen to be a part of the 
IAPT institution that was responsible for his alienation, but by adopting this rebellious stance in opposition to her, he avoided direct confrontation with his IAPT placement.

\section{Scientific Approach Discourse}

A scientific approach discourse seemed to emerge in those instances where participants appeared to draw on an empirical epistemology, where phenomena are spoken of as things that can be measured and recorded through systematic observation and subjected to certain principles of reasoning. The following extract shows how a participant drew on this discourse when she was asked the question 'So what is IAPT?' in her interview:

...oh IAPT it's just a system it's a black box... (Anne).

According to Kendall and Wickham (1999: 73), a 'black box' is a term that is used in science or engineering 'when something is too complex to be fully explained or represented'. They cite the work of Latour (1987) who argued 'that when something has been "black-boxed", it is simply used in a chain of arguments' without elaboration. Through the discourse of a scientific approach, this participant constructed IAPT as a 'system' that is unknowable, or too complex to be defined. Her use of this discourse appeared to position her as someone who did not know much about IAPT and perhaps did not want to. The description closed down the conversation, instead of trying to open up what might be inside 'the black box'. It could be interpreted that this construction of IAPT limits the action that this 
participant can take, therefore limiting her personal power and ability to construct any knowledge about IAPT.

The following extract demonstrates where a participant attempted to draw on a scientific approach discourse to question the influence that a 'scientific' way of thinking has on the way in which we acquire knowledge:

...it's a great challenge to try and offer knowledge (2) in a different way that isn't scientific (2) our culture is too caught up in cause and effect... (Dee).

When asked by the interviewer what she meant by this, she continued:

...as therapists we don't have to get caught up in it but if we want to be part of the (4) I can't find the word um (.) evidence-based kinds of therapy that IAPT loves so much (.) we then need to produce our own evidence (9) oh I don't know I'm lost [laughs]... (Dee).

In this extract Dee struggled to find words. Although it appeared that she was trying to use a scientific approach discourse to speak her views against it, she became caught up in the very issue that she believed 'our culture' gets caught up in, which appeared to reflect the difficulty of how hard it was to criticise this discourse from within the discourse. Before she was able to come to any conclusions, she invalidated what she had said by stating 'I don't know I'm lost', as if she was not sure what she was supposed to say, or what could be said from this position 


\section{Crossing Discourses}

Two final discourse categories are presented here under the subheading 'crossing discourses', representing the instances where certain discourses appeared to emerge simultaneously, yet in opposition to each other.

\section{Non-IAPT discourse vs IAPT discourse}

A non-IAPT discourse seemed to emerge in those instances where participants discussed their training background and their 'private practice' or 'own practice'. This seemed to contrast the way in which they felt they had to 'work' within an IAPT setting, which seemed to draw on what has been described here as an IAPT discourse.

In the following extract, Anne described the psychodynamic way in which she approached her own practice:

...the way I like to be in my /own/ practice (.) well it's more about the client really and the relationship he has with me (2) if he wants to work deeply if he can manage the emotion if he can establish a trust with me (.) it can be a very intense relationship [] and very rewarding... (Anne).

She constructed her 'own practice' by drawing on a discourse that privileges the relationship established between herself and her client through talking. She 
appeared to take up the subject position of confident advocate of her own practice and talked of the establishment of such a relationship as 'rewarding' experience. When the interviewer went on to ask her to describe her experience of client relationships at the IAPT service, she was no longer able to maintain this discourse:

...it's like a conveyor belt (.) ticking boxes with the clients (.) data and graphs are generated on the computer [ ] in supervision we tick more boxes (.) we're dealing with a system rather than people... (Anne).

She had not had any additional IAPT-accredited training, and yet the way in which she worked with her IAPT clients is spoken of in a different way. 'Ticking boxes' appears to construct the IAPT service as something mundane or routine; a complete contrast to what she said about the 'intense relationship' that can be established in client work.

In the following extract, Claire had been asked to describe her training background and approach to counselling and responded:

...there's something about when you're working with a client that you get to almost a different level of communication (2) I can't really describe what this level is but I kind of know it when it's happening [ ] there's kind of a (.) intuition or some connection [ ] in my private practice this is what (.) happens... (Claire). 
She immediately continued, unprompted, discussing her role as an 'IAPT psychological therapist':

...but I find that when I'm working with clients in this (2) system that this is harder to reach (.) because there's always an awareness that I've got to follow certain rules (.) certain protocol... (Claire).

In this extract she appeared to draw on a language that referred to 'rules' and 'protocol'. The use of this discourse does not make available the same subject position as when she had been describing her 'private practice', but rather she assumes the subject position of compliant worker who follows the 'rules' and 'protocol', contradicting her previous description of client work. Again, it could be interpreted that this might be a similar example to that of Anne, where opposing subject positions and conflicting feelings have been made available at the discursive junction of an IAPT and non-IAPT discourse.

The following extract shows how Dee believed that she was able to work in the existential approach of her training within her IAPT placement:

...I tell my IAPT clients that I work Existentially [ ] but I wonder if I don't shout about that outside the room [ ] it's almost like [lowers voice] I'll do it but I won't tell anyone (2) because I might be told [raises voice] no no no you should be doing it our way(.) the IAPT way... (Dee). 
The subversive subject position she appeared to take up at first seemed to suggest that Dee was working the way she wanted (Existentially), but then she admitted that she felt like she had to do so in secret. It could be interpreted that the feelings made available by this subject position included anxiety and fear about being discovered, as indicated by her lowered voice, and a non-IAPT discourse is 'hushed' to alleviate anxiety.

\section{Psychological vs Medical Power}

It was noted how, in some cases, language was used that appeared to construct a development towards 'psychological wellbeing' in clients, while attempting to draw on concepts such as 'diagnosis and treatment' that might belong to the medical system. This seemed to contrast the way in which participants positioned themselves outside of this system.

In the following extract a participant talks about her use of IAPT weekly questionnaires:

...the computer plots a graph week by week and you've got a blue line and a red line and the red line is [ ] indicating if there is any risk (.) it's bad if they dip into the at risk area yeah (.) and the blue line measures psychological wellbeing and health... (Claire).

Claire was discussing a graph that could be viewed on a computer screen, once the weekly IAPT questionnaire data had been typed into the computer system. She 
seemed to draw on medicalised language to indicate that she viewed 'psychological wellbeing and health' as things that could be measured and quantified by the graph. She went on to state:

....and so it kind of fluctuates from week to week and you can see when it's going up or down and this area's on the graph where it goes from healthy to [ ] severe distress... (Claire).

Claire had stated earlier in the interview that the 'graph' was comprised of numerical values only, yet she chose to name the opposite ends of the graph 'severe distress' and 'healthy'. Through the use of such language, she appeared to be demonstrating her co-operation with using the IAPT computer programme to measure her client's progress between these two opposite points of the graph, but when she was asked by the interviewer what these data were used for she replied:

...they [IAPT] can detect from the data whether or not the client is getting better or worse... (Claire).

Instead of using the personal pronoun 'l', Claire said 'they can detect', positioning herself as excluded from the process of measuring the client's 'psychological wellbeing and health'. It could be argued that the way in which she crafted her story (that she is compliant with using the computer system, but does not include herself in 
using the data to measure her client's performance) made available feelings of being excluded from, or an outsider to, the IAPT service.

The participant in the following extract spoke about her experience of working as an IAPT practitioner in the setting of a GP surgery:

...I work in a room upstairs (.) but once I had a disabled client and had to borrow a GP's consulting room on the ground floor [ ] while I was seeing that client I was moved around week to week and had to make do with whatever room was available... (Emma).

While she had described where she worked upstairs as a 'room', a similar room downstairs was spoken of as a 'consulting room', because of the association with the GP. She continued by describing the following incident in a session with the same disabled client:

...we were mid-session and this GP just waltzed in (.) she hadn't been told that I was using her room (.) she was s:::o angry and I just kept apologising for being there even though the practice manager had OK'd it (4) argh I still feel embarrassed just thinking about it [covers face with hands]... (Emma). 
The way in which Emma crafted her account of events implied that the confusion might have been a fault on the part of practice manager or GP and her use of the term 'waltzed in' carries the implication that the GP was able to do whatever she liked. Yet the discourse that Emma employed seemed to position her as if she was in the wrong, or imposing, and that she was somewhere she should not have been. It could be interpreted that feelings of embarrassment have been made available by the subject position that she has assumed of imposter - of someone discovered in a place they should not be - because it seemed that from this subject position, the authority of the GP cannot be questioned.

\section{Reflexivity}

As part of personal reflexivity, my role in the study and how this may have influenced the outcome was considered. Mills (2003: 41) cites Kendall and Wickham (1999:51) that Foucauldian Discourse Analysis should aim to 'describe the way in which resistance operates as a part of power, not seek to promote or oppose it'. However, as Mills points out, the very choice of the discursive object of analysis already 'suggests a particular position in relation to which side in a conflict one is supporting' (2003: 41) and as a non-IAPT counsellor working in an IAPT service, I had already taken up a position in terms of my research question. My personal experience as a therapist struggling to integrate her core theory with working in an IAPT context, meant that I had already created the subject position of 'non-IAPT' therapist before I had even set out to investigate what other therapists had to say about the subject. This struggle was intensified when permission to recruit participants through my IAPT placement was ignored. I had to confront my own angry feelings and decide 
whether to 'cease and desist' or take my research elsewhere. In doing that, while continuing to work at the placement, again, another subject position was taken up and I cannot deny the possibility that this research paper may have become a means to regain power in a situation in which I had felt powerless.

If one of the initial aims of the study was to look for ways that power could be constructed by discourses within IAPT, the power dynamic set up in the interview situation cannot be ignored. Kvale \& Brinkmann (2009: 33 - 34) discuss power asymmetry in regard to the qualitative research interview process, in particular that the interview contains mainly one-way dialogue that can be both instrumental and manipulative. The interviewer has monopoly of interpretation over the participants' statements, with 'exclusive privilege to interpret and report what the interviewee really meant' (Kvale \& Brinkmann, 2009: 34). It therefore has to be taken into consideration that different results may have emerged if someone else had conducted the interviews.

Another important consideration is that it was already known to all participants, before the interviews took place, that I was also a 'non-IAPT' therapist working for an IAPT service. Although it was initially assumed that this might allow the participants to feel more in an 'equal' position, it became apparent through the difficulty of finding participants and the number of participant withdrawals that this might not have been the case. It is a possibility that my perceived position of 'IAPT insider' or 'IAPT representative' meant that some of those who had been approached would be reluctant to participate and cautious not to say 'too much' and it is equally possible that participants were censoring themselves and might have just been saying what they thought they were supposed to be saying, or what they thought was expected of them. 


\section{Discussion}

In what was said, IAPT was constructed through different discourses as a powerful service provider. Participants appeared to draw on what were seen to be 'dominant discourses', in attempts to fit in or belong to IAPT, and it was considered that perhaps these dominant discourses could fall under the umbrella of 'IAPT discourses'. At the same time, however, a paradox emerged because the way in which these discourses were used by participants seemed to create subject positions that were outside to, or excluded from, the IAPT system. In turn, such positions did not allow much room for any kind of action to be taken, except to comply with the system, and this may have served to legitimise feelings of powerlessness.

It seemed that when using these dominant discourses or IAPT discourses, the description of counselling and psychotherapy as a human endeavour was lost and there seemed to be no space to talk about relationships or people. It was as if the discursive constructions of IAPT as 'a programme', 'a system', or a 'black box' had dehumanised therapist-client interaction to such a degree, that there was no room left to think about counselling in terms of human relationships, and clients could only be spoken about in a detached way, or not mentioned at all. Rizq (2012: 21) suggests that such attitudes from those who work in public services may come about 'because the invisible, reparative aspects of psychological care - the emotional contract between patient and therapist - are deemed invalid where they do not directly contribute to the measurable output of a service'. 
Conversely, the same participants seemed comfortable discussing their 'relationships' with their clients, when discussing their 'private practice' or 'own practice'. This 'non-IAPT discourse' appeared to allow for the positioning of each participant as a confident advocate of their own approach to counselling and/or psychotherapy. However, participants only seemed able to maintain such positions by distancing themselves from IAPT and making a separate reference to 'private' or 'own' practice. When a non-IAPT discourse was drawn on in this way, the IAPT discourse seemed to be subverted. It was considered how there appeared to be a split in the subjectivity of each participant and that this positioning might allow a particular 'self' to step in. It could be argued that by avoiding speaking this nonIAPT discourse when referring specifically to their IAPT placements, the participants were able to justify their position of 'IAPT psychological therapist'. Likewise, by avoiding speaking in an IAPT discourse in certain instances, participants were able to justify their positions of 'Existential Therapist', 'Person-Centred Therapist' etc. Such instances, where two opposing world views appeared to be coming up against each other, were thought to demonstrate the crossing of discourses (or discursive junctions) occurring when contradictory ideological discursive resources were drawn upon.

It was noted that a discursive junction also seemed to occur when 'psychological power' came up in opposition to 'medical power'. IAPT (and the psychological therapies it represents) seems to fit within the NHS and its commitment to 'health' and 'well-being', yet as soon as it is placed within the setting of the NHS, it has to be 'medicalised' and becomes something 'other' than that what was originally intended. In some instances participants seemed aware of this dichotomy and appeared to hesitate, or even resist, using certain discourses. Parker 
(1992: 10) asks the question 'what right do we have to speak in a discourse?' to demonstrate how we are 'positioned in a relation of power when we are placed in relation to the discourse itself'. During the interviews it appeared that whether participants felt they had a right to speak in the discourse or not, ultimately the use of discourses cannot be avoided.

\section{Limitations}

The findings and subsequent interpretations presented above are not assumed to be pre-existing. As Foucault once stated:

\footnotetext{
we must not imagine that the world turns towards us a legible face which we would only have to decipher; the world is not the accomplice of our knowledge; there is no pre-discursive providence which disposes the world in our favour (in Young, 1981: 67).
}

Although the findings are a representation of some of the things that were spoken about by participants during their interviews, it is recognised that the moment certain extracts were privileged and included in the study, it meant that others had to be excluded. By the same token, discourse analysis not only considers what is said, but also what cannot be said, or what is impossible to say, from within a certain discursive location. Questions arose about what the participants did not say, in order to maintain their subject positions. In terms of the recruitment process, it is considered that this may have contributed to the difficulty in recruiting participants 
and provides a possible explanation as to why it appeared that nobody wanted to talk about their experience of working within IAPT services.

As a part of personal reflexivity, questions were raised throughout the study as to whether the researcher should try to step outside of the institution of IAPT in order to study it. Foucault's descriptions of power seem to assume that we can never step outside of it to provide an objective analysis of it, so one has to exploit the position from within. Yet in doing this, it became apparent that my own subject position may have influenced my recruitment, interview process and the analysis of my results. My role as a 'non-IAPT' therapist working for an IAPT service had been made known to all the participants and therefore the views that participants expressed may well have been in an effort to please me. In other words, they may have been telling me what they thought I wanted to hear.

The present study can be criticised for the narrow focus of people interviewed. It is suggested that perhaps a future study should be conducted to examine whether IAPT trained practitioners might exhibit such dissonance too; in the difference between how they believe they are required to work and how they see themselves actually working. If such a study produced similar findings, then one might speculate that there must be something about the construction attributed to an IAPT service that produces this dissonance, rather than the non-IAPT status of some practitioners.

While outside the scope of this paper, questions have emerged regarding the implications for quality of care and in turn, client outcomes, when therapists are working with therapy models to which they have no allegiance. This seems to open up another area that could be further explored by future research. 


\section{Conclusion}

As IAPT continues influence how psychological therapy is made available within the NHS, this study aimed to explore the relations of power that may be in operation within this setting, from the perspective of non-IAPT trained counsellors and psychotherapists. Overall it is suggested that the IAPT service appeared to be given power by the way in which it was constructed by participants, through the use of dominant discourses in their attempts to fit in or belong to the service. Paradoxically, however, the use of these discourses only served to create subject positions that were outside to, or excluded from, the IAPT system. Instances where two opposing world views appeared to be coming up against each other were thought to be examples of the discursive junction, where opposing discourses could not be spoken simultaneously. The findings highlight that where the dominant discourses of IAPT were used, the description of counselling and psychotherapy as a human endeavour was lost and there seemed to be no space to talk about relationships or people. The only way participants seemed able to do so was to revert to speaking of their 'private practice' or their 'own practice'. This may hold important implications in particular for IAPT about the ideological effects of power and other possible research studies have been suggested. These include an exploration in to whether IAPT trained therapists may exhibit such dissonance too; in the difference between how they believe they are required to work and how they see themselves actually working, and also the implications for quality of care and client outcomes when therapists are working with therapy models to which they have no allegiance. It is hoped that further research studies such as these will keep us all asking questions about a psychological therapies service that seems set to stay around for a while longer - instead of trying to 'black box' it as something that is too complex to be spoken about. 


\section{REFERENCES}

Clark, D.M., Layard, R., Smithies, R., Richards, D.A., Suckling, R. and Wright, B. (2009) 'Improving access to psychological therapy: Initial evaluation of two UK demonstration sites'. Behaviour Research and therapy, Volume 47, Issue 11, November 2009, Pages 910-920.

Clark, D.M. (2011) 'Implementing NICE guidelines for the psychological treatment of depression and anxiety disorders: The IAPT experience'. International Review of Psychiatry, 23: 318-327.

Cooper, M. (2011) Development of a randomised controlled trial of counselling for depression. Working paper. British Association for Counselling \& Psychotherapy, Lutterworth. (Unpublished) Accessed via http://strathprints.strath.ac.uk/id/eprint/30518 [date last accessed 10.4.2013].

Foucault, M. (1972) The Archaeology of Knowledge (trans. A.M. Sheridan Smith). London: Routledge.

Foucault, M. (1994). 'An Interview with Michel Foucault'. In J. D. Faubion (Ed.), Power (Vol. 3, pp.239-297). New York: The New Press.

Guy, A., Loewenthal, D., Thomas, R. and Stephenson, S. (2012) 'Scrutinising NICE: The impact of the National Institute for Health and Clinical Excellence Guidelines on the provision of counselling and psychotherapy in primary care in the UK'. Psychodynamic Practice, Vol. 18, No. 1, 25-50.

Health and Social Care Information Centre (HSCSC) (2009) Adult Psychiatric Morbidity in England - 2007: results of a household survey. Leeds. Acessed via http://www.hscic.gov.uk/pubs/psychiatricmorbidity07 [last date accessed 17.06.13]

Kendall, G. and Wickham, G. (1999) Using Foucault's Methods. London: Sage.

Kvale, S. \& Brinkmann, S. (2009) Interviews: Learning the Craft of Qualitative Research Interviewing $\left(2^{\text {nd }} \mathrm{Ed}\right)$. London: Sage.

Latour, B. (1987) Science in Action: How to Follow Engineers in Society. Milton Keynes: Open University Press. In G. Kendall and G. Wickham (1999) Using Foucault's Methods. London: Sage. 
Layard, R. (2005) Mental Health: Britain's Biggest Social Problem. LSE Research Online. Accessed via:

http://cep.Ise.ac.uk/textonly/ new/staff/layard/pdf/RL414 Mental Health Britains Bi ggest Social Problem.pdf [last date accessed 10.4.2013].

Loewenthal, D. and Winter, D. (2006) What is Psychotherapeutic Research? London: Karnac.

McLeod, J. (2001) Qualitative Research in Counselling and Psychotherapy. London: Sage.

McLeod, J. (2011) Qualitative Research in Counselling and Psychotherapy (2 $\left.{ }^{\text {nd }} E d\right)$. London: Sage.

Mills, S. (2003) Michael Foucault. Oxon: Routledge.

NICE (2013) Accessed via: http://guidance.nice.org.uk/ [last date accessed 10.4.2013]

Orwell, G. (1949) Nineteen eighty-four. London: Secker \& Warburg.

Parker, I. (1992) Discourse Dynamics: Critical Analysis for Social and Individual Psychology. London: Routledge.

Parker, I. (2005) Qualitative Psychology: Introducing Radical Research. Berkshire: Open University Press.

Parker, I., Georgaca, E., Harper, D., McLaughlin, T. and Stowell-Smith, M. (1995) Deconstructing Psychopathology. London: Sage.

Potter, J. (2004) 'Discourse analysis as a way of analysing naturally-occurring talk'. In D. Silverman (Ed) Qualitative Research: Theory, Method and Practice $\left(2^{\text {nd }} \mathrm{Ed}\right)$. London: Sage.

Risq, R. (2012) 'The perversion of care: Psychological therapies in a time of IAPT'. Psychodynamic Practice: Individuals, Groups and Organisations, 18:1, 7-24.

Sarup, M. (1993) An Introductory guide to Post-Structuralism and Postmodernism ( $2^{\text {nd }}$ Edition). Athens: University of Georgia Press.

Silverman, D. (2004) (Ed) Qualitative Research: Theory, Method and Practice (2 ${ }^{\text {nd }}$ Ed). London: Sage. 
Silverman, D. (2006) Interpreting Qualitative Data ( $3^{\text {rd }}$ Edition). London: Sage.

Willig, C. (2008) Introducing Qualitative Research in Psychology. Berkshire: Open University Press.

Young, R. (1981) Untying the text: A Post-structuralist Reader. London: Routledge, Kegan \& Paul. 